\title{
STUDY OF THE OPERATION OF THE CORNELL LINAC FOR POSITRON PRODUCTION AT HIGH MULTIBUNCH CURRENTS*
}

\author{
V.S. Alexandrov, A.P. Ivanov, N.Yu. Kazarinov, E.A. Perelstein, M.N. Sazonov \\ JINR, Dubna, Russia, 141980 \\ M.G. Billing \\ Cornell University, LNS, USA, 14583
}

\section{Abstract}

The Cornell Electron Storage Ring (CESR) operates as an electron-positron collider with 9 trains of 2 to 5 [1] closely spaced bunches per train per beam. The Cornell Linac is the initial component of the injector for CESR and it needs to provide multibunch trains of electrons and positrons at high injection rates for CESR. This paper presents the results of simulations which have studied the effects of operating the Linac at high multibunch currents needed for the production of trains of positron bunches. Results include the effect of longitudinal wakefields induced by the beam in the linac injector prebuncher cavities and a method for the partial compensation of the effect of these wakefields, the effect of transverse wakefields in the linac accelerator sections in the electron linac preceding the positron target and the effect on positron capture of increasing the accelerating gradient in the positron linac. Comparison with some linac measurements is included.

\section{WAKEFIELDS IN THE PREBUNCHER CAVITIES OF THE LINAC INJECTOR}

The Cornell linac injector creates a beam of $300 \mathrm{MeV}$ electrons or a beam of $200 \mathrm{MeV}$ positrons. The positrons are produced in a tungsten target on the converter installed in the middle of the linac. The linac is modelled with eight SLAC accelerating sections AS\#1...AS\#8 [2]. The positron conversion efficiency is about $0.1 \%$. Such a small yield requires the highest current of the electron beam during positron production. Thus the largest current is in the initial part of the injector. The first half of the linac consists of the $150 \mathrm{keV}$ electron gun, two identical prebuncher cavities operating at $214 \mathrm{MHz}$ and the first linac sections AS\#1...AS\#4.

The linac injector provides a high current electron beam with 7 trains of 2 to 5 bunches accordingly to the CESR operation described above. In the case of positron production each bunch is formed from 3 ns gun pulse with $1.4 \cdot 10^{11}$ electrons. These electron pulses excite the wakefields synchronously on the fundamental mode of the prebuncher cavities. The accumulated longitudinal wakefields decelerate the bunches, distort their bunching and reduce the number of particles captured into the linac.

A special method for the partial compensation of these wakefields is used for the CESR injector. The gun introduces an additional delay $\tau$ between the electron bunches. As a result the bunches are shifted at the accelerating phase of RF voltages of the prebuncher cavities. Thus the decelerating effect of the wakefields is compensated by the RF field. For example, the simulations of the net voltages (RF bunching voltage and wake potential) for the $14^{\text {th }}$ bunch are shown in Fig. 1 for the gun using compensated and uncompensated timing parameters.

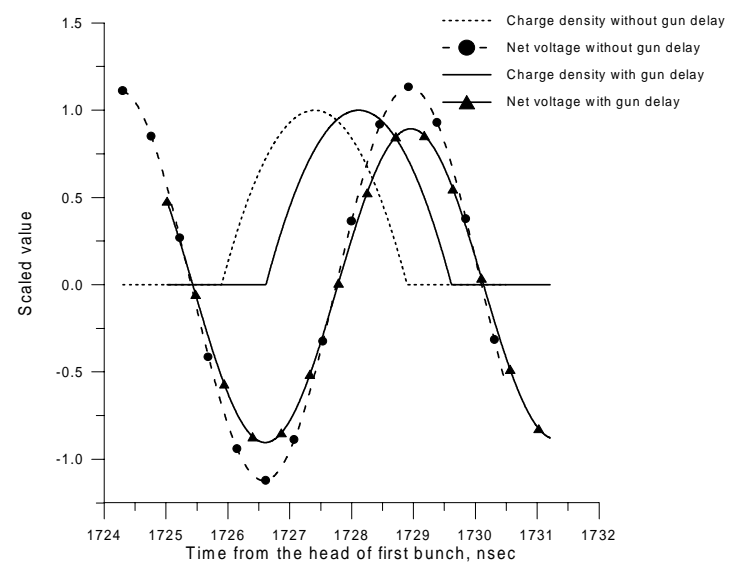

Figure: 1 Net bunching voltage in the first prebuncher.

The wakefields have been simulated for 7 trains of 2 bunches. Two cases of different RF bunching voltages $35 \mathrm{kV}, 105 \mathrm{kV}$ and $50 \mathrm{kV}, 150 \mathrm{kV}$ have been studied for first (PB\#1), second (\#PB2) prebuncher cavities correspondingly. The wake potentials have been obtained in terms of the cavity mode data calculated by the URMEL code. The quality factor $Q$ at the fundamental mode is high enough to neglect attenuation of the wakefields during the traversal by the entire beam ( 7 trains by $280 \mathrm{~ns})$. The wakefield amplitudes in the both cavities are proportional to the number of bunches which

* This work has been carried out under NFS contract No. PHY-9310764 SUB U82-8446. 
passed earlier. The bunching effect in the drift space between the prebuncher cavities squeezes the bunch length. As a result the wakefields have amplitude in 1.2 times higher in the second prebuncher in comparison with the first one. The value of delay $\tau$ is chosen from the condition of the wakefield compensation. The result of simulations gives the relative time between the centroids of the bunches [difference in the injection phases of RF linac $\left(1 \mathrm{psec} \leftrightarrow 1^{0}\right)$ between bunches] are shown in Fig.2.

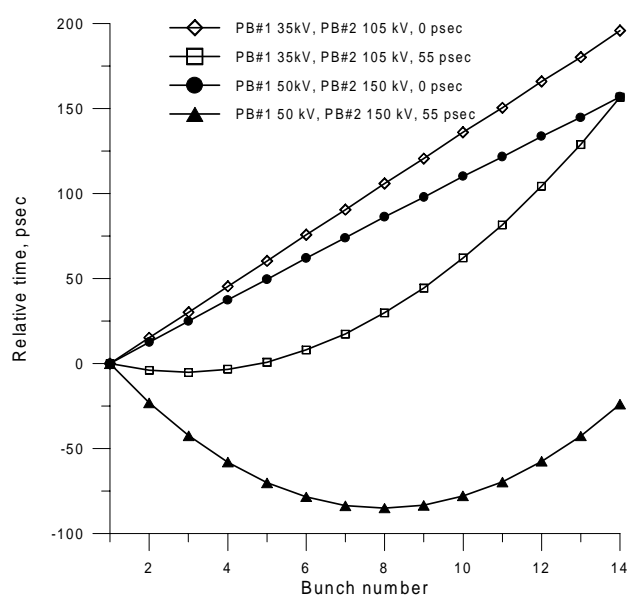

Figure: 2 The relative time between bunches at the linac entrance.

The special gun timing correction provides more successful injection of the high current electron beam into the linac. It is possible to get an optimal case for $14^{\text {th }}$ bunch structure providing $22.5 \mathrm{nC}$ charge per bunch by tuning the RF prebuncher voltages and the gun delay $\tau$. Using the delay $\tau$ also provides the maximum current of electron beam for operation with 7 trains per 5 bunches. In this case the optimal charge per bunch is estimated as $11 \mathrm{nC}$ approximately.

\section{TRANSVERSE WAKEFIELDS IN THE LINAC}

The linac of the CESR injector is modelled to contain SLAC accelerating sections. The experimental measured frequency spectrum of the dangerous dipole modes extends from 4143 to $4350 \mathrm{MHz}$ [3] approximately. The amplitude of the dipole wakefields is proportional to beam displacement away from the electrical center of the linac sections. From estimations of the linac optics we put this displacement to $1 \mathrm{~mm}$ at the entrance into the sections. The wakefield model [4] has been developed for constant gradient structures. It assumes the accelerating section consists of a collection ( $N=86$ cells) of uncoupled oscillators corresponding to the synchronous modes of the periodic structures. Thus the wake potential after the bunch number $\mathrm{p}$ is

$$
W_{p}(t)=\frac{1}{N} \sum_{j=1}^{p} \sum_{i=1}^{N} W_{T, i}\left(k_{T, i}, \omega_{i}, r_{q i}, Q_{i}, t+t_{j p}\right),
$$

where $k_{T}[\mathrm{~V} / \mathrm{C}]$ and $r_{q}$ are the loss factor and the displacement of an exciting charge, $\omega$ and $Q$ are the angular frequency and the quality factor of the dipole mode, $i$ and $j$ are cell and bunch indexes, and $t$ is time between bunches.

There is solenoid focusing in the first accelerating section AS\#1 of the linac with the longitudinal magnetic field $B=0.2 \mathrm{~T}$. Simplified assumptions of the bunch motion shows the final beam displacement at the exit of the section should be about $1.1 \mathrm{~mm}$ due to solenoid focusing. The wakefields and the bunch motion are calculated in each cell for the second linac section AS\#2 which has no solenoid coils.

The bunch to bunch influence depends strongly on the frequency of the dipole modes. In this case it is possible only to determine the worst situation when, due to the wakefields, one of the given bunches gets the maximum displacement after the linac section. We solve the equations of motion changing the frequency of the first cell of the accelerating section and keeping the frequency detuning range up to $5 \%$ for the last section cell. The bunch displacements for the "worst" situation are shown in Fig. 3. Time between bunches in the train is $14 \mathrm{~ns}$ and time between trains $280 \mathrm{~ns}$. The charge of every bunch is $22.5 \mathrm{nC}$.

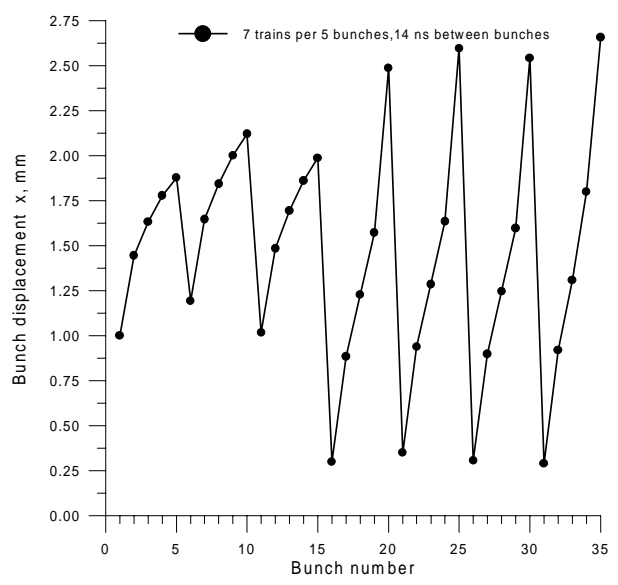

Figure: 3 The bunch displacement after AS\#2.

The influence of the wakefields in the next sections is less due to the higher beam energy. It is possible to provide the high multibunch current in each linac sections. However the instability from bunch to bunch coupling should be studied in more details for the whole linac.

\section{IMPROVEMENTS IN THE POSITRON LINAC USING HIGHER ACCELERATING GRADIENTS}

The simulations of the positron dynamics have been performed assuming the possibility that the input RF power in the accelerating sections \#5 and \#6 may be increased from $15 \mathrm{MW}$ up to $24 \mathrm{MW}$ to enlarge the positron transfer through the positron linac. The electrons passing through the converter target and secondary 
electrons from the conversion process are accelerated in these sections and contribute sufficiently to the net observed charge. The CESR measurements [5] have shown that the total beam charge is negative during acceleration up to the AS\#6 where it changes the sign. To have similar results in the simulations we searched for a regime with such effect. In the case of the maximum energy gain for positrons the total beam charge (the difference between the numbers of electrons and positrons) does not change sign during acceleration in the positron linac.

To find out the parameter set which gives this same change in sign of the net accelerated charge, the starting phase (WT0) with the fixed RF field phases of accelerating sections of the positron linac have been variated. Results of the calculations are shown in Fig. 4. The total charge after AS\#6 changes sign in a narrow region of the starting phases. Unfortunately the energy gain in this region is very small, i.e. the regime with changing of the charge sign is not optimal to obtain the maximal energy of positrons. To increase the final positron energy to about $200 \mathrm{MeV}$ [6] the appropriate RF phases of AS\#6 - AS\#8 have been chosen. As result the total charge of the beam becomes near zero after AS\#6.
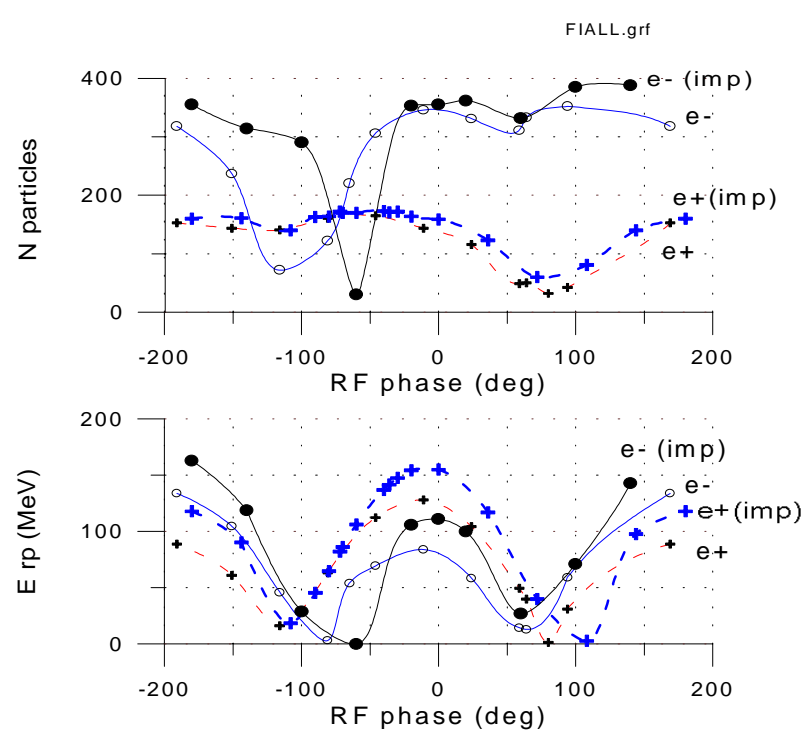

Figure: 4 Number of particles and the reference particle energy at the end of AS\#6.

The tuning of the quad gradients was made to maximize the energy spectrum of the beam. Fortunately the gradients in quads between AS\#5 - \#6 and AS\#6 - \#7 do not significantly influence on the positron current at the end of AS\#8. The gradients of the triplet between AS\#7 \#8 noticeably affect the positron transmission.. For example, the changing of the gradients on the value of $100 \mathrm{G} / \mathrm{cm}$ remove the positron losses in AS\#8.

To estimate the efficiency of the new klystron installation the new (improved) case was simulated. This new variant differs from existing one by improved accelerating gradient in AS\#5 and \#6, correspondingly tuned lenses gradients and bending magnet field, and decreased on $10 \%$ the magnet field of AS\#6 solenoid.

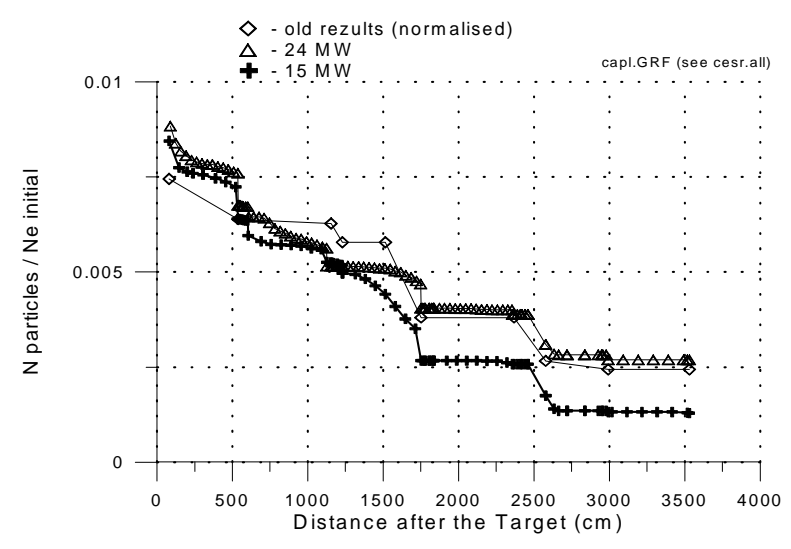

Figure: 5 Positron transmission through the positron linac.

The comparison of the simulation results for the input RF power $15 \mathrm{MW}$ and $24 \mathrm{MW}$ is shown in Fig. 5. It can be seen that the positrons yield at the end of the positron linac has increased about $70 \%$. Additionally the average positron energy gain increases about $20 \%$ : from $200 \mathrm{MeV}$ to $235 \mathrm{MeV}$. The summary plot of the total beam charge transmission through the positron linac and the dipole magnet is shown in Fig. 6.

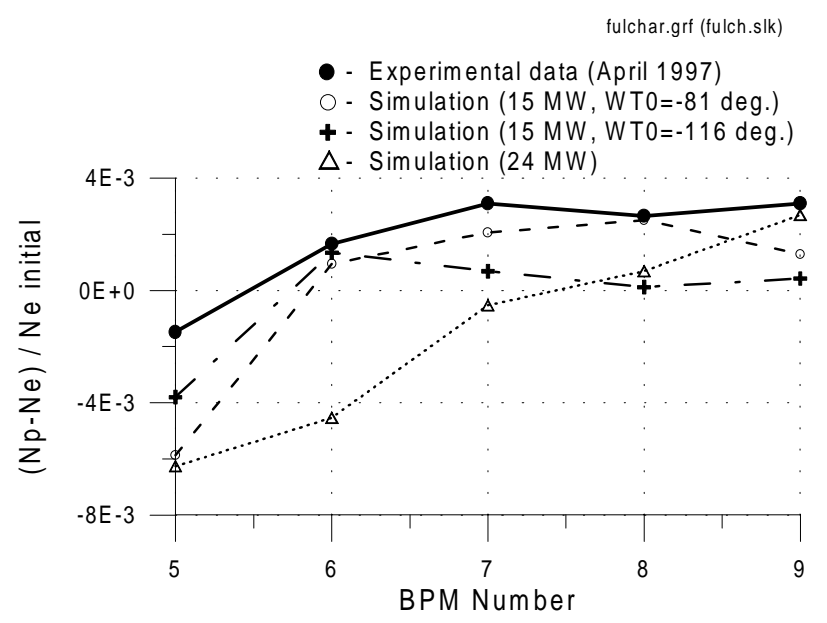

Figure: 6 Total charge transmission through the positron linac.

\section{REFERENCES}

[1] D.Rise, Proc. of EPAC96, Barcelona, 1996, p.17.

[2] K. Berkelman et al., «CESR-B Conceptual design for a B Factory based on CESR», Cornell Univ., CLNS 91-1050.

[3] M.Seidel, et al., SLAC-PUB-7557, May, 1997.

[4] K. A. Tompson, et al, SLAC-PUB-6032

[5] M.Billing, M.Sazonov, Cornell Univ., LNS, Private communication, April, 1997.

[6] R.Cutler, Cornell Univ., LNS, Private communication, September, 1996. 